\title{
A percepção do cuidador familiar acerca do brincar de crianças em vigilância do desenvolvimento em um serviço de terapia ocupacional no estado do Pará
}

\author{
The familiar caregiver perception about child playing in development vigilance in a \\ occupational therapy service at state of Pará
}

La percepción de los cuidadores familiares sobre jugar niños en la vigilancia del desarrollo en un servivio de terapia ocupacional en el estado de Pará

Daniel Corrêa Coimbra ${ }^{1 *}$, Fernanda Désirée Teles Monteiro ${ }^{1}$, Aliane Natalia Bahia Botelho ${ }^{1}$, Karina Saunders Montenegro'.

\section{RESUMO}

Objetivos: Compreender a percepção do cuidador familiar acerca do brincar de crianças em programas de vigilância do desenvolvimento. Métodos: Abordagem qualiquantitativa, de natureza descritiva e exploratória. O estudo ocorreu na Escola Superior da Amazônia - ESAMAZ, no projeto de vigilância do desenvolvimento ACRESCER, após critérios de inclusão e exclusão participaram quatro cuidadores familiares. A análise dos dados ocorreu pela interpretação e atribuição de significados dos principais dados obtidos a partir da análise de conteúdo. Resultados: $75 \%$ dos cuidadores são mães, com renda acima de quatro salários mínimos, e $75 \%$ com nível de escolaridade de ensino superior completo. A idade das crianças acompanhadas variou em um intervalo de um ano e seis meses à seis anos. Os dados qualitativos foram organizados em quatro eixos temáticos, o tempo da brincadeira, o brincar com outras crianças, o brincar e o desenvolvimento da criança, e a participação do cuidador durante o brincar. Conclusão: Os cuidadores familiares consideram suficiente o tempo de duração do brincar, apresentam a percepção da brincadeira como fonte importante para 0 desenvolvimento infantil e como principal ocupação da criança, acreditam que o brincar contribue no favorecimento do vínculo familiar e o consideram importante para o desenvolvimento das relações sociais.

Palavras-chave: Desenvolvimento infantil, Cuidador familiar, Terapia ocupacional.

\begin{abstract}
Objectives: Understand the family caregiver perception about playing children in vigilance development program. Method: Quanti qualitive aproach with a descriptive and explanatory nature. The research occurred in Escola Superior da Amazonia - ESAMAZ, in a vigilance development project ACRESCER, after some inclusion and exclusion requirements, four familiar caregivers participated. The data analysis occurred through the attribution and interpretation of meanings main obtained data starting content analysis. Results: $75 \%$ of caregivers are mothers, with an income above four wages, and $75 \%$ with complete higher education level. The accompanied children ages varied in a break of one year and six months to six years. The qualitative data were organized in four thematic axes, the playing time, the playing with another children, the playing and the children development and the caregiver participation during the play. Conclusion: The familiar caregivers consider the time duration to play as enough, and to present the playing perception as a important source of child development like a main child occupation, believe that playing contributes to favoring the family bond consider it important to development of social relationship.
\end{abstract}

Keywords: Child development, Familiar caregiver, Occupational therapy.

${ }^{1}$ Escola Superior da Amazônia (ESAMAZ), Belém - PA. *E-mail: dancoimbra83@gmai.com

SUBMETIDO EM: 6/2020 ｜ＡCEITO EM: 7/2020｜ＰUBLICADO EM: 10/2020 


\section{RESUMEN}

Objetivos: Comprender la percepción de los cuidadores familiares sobre jugar con niños en programas de vigilancia del desarrollo. Método: Enfoque cualitativo e cuantitativo de naturaleza descriptiva y exploratoria. Estudio se realizó en la Escuela Superior de Amazonia - ASAMAZ, en el Proyecto de vigilancia del desarrollo ACRESCER, después de los criterios de inclusión y exclusión participaron quarto cuidadores familiares. EI análisi de los datos ocurrió a través de la interpretación y atribución de significados de los principales datos obtenidos a partir del análisi del argumento. Resultados: $75 \%$ de cuidadores son madres con un ingreso superior de cuatro salarios mínimos, y el $75 \%$ con un nivel de educación superior. Las edades de los niños acompañados varió entre un a seis meses. Los datos cuantitativos fueron organizados en cuatros ejes temáticos, tiempo de juego, jugar con otros niños, juego y el desarrollo de niños, y la participación de cuidadores durante el juego. Conclusión: Los cuidadores familiares consideran que el tiempo de duración de juego es suficiente, presentan la percepción del juego como una fuente importante para el desarrollo infantil y principal ocupación de los niños. Creen que el juego contribuye a favorecer el vincula familiar y consideran importante para el desarrollo de las relaciones sociales.

Palabras clave: Desarrollo infantil, Cuidadores familiares, Terapia ocupacional.

\section{INTRODUÇÃO}

A infância, enquanto a primeira fase da vida compreende um período de significativas modificações no desenvolvimento neuropsicomotor, exigindo acompanhamento regular da criança, a fim de detectar precocemente possíveis alterações. Este acompanhamento deve iniciar desde o nascimento, pois os dois primeiros anos de vida constituem-se em um período significativo e ideal para intervenções que previnem ou tratem problemas no desenvolvimento (FIGUEIRAS AC, et al., 2005).

No Brasil, o acompanhamento do desenvolvimento de crianças nos primeiros anos de vida ocorre através dos programas de vigilância do desenvolvimento. O profissional de saúde que atua nestes programas deve aplicar instrumentos de avaliação para detectar possíveis atrasos no desenvolvimento infantil, identificando as possíveis causas, orientando os pais para que estes auxiliem e estimulem a criança (FIGUEIRAS AC, et al., 2005). Neste contexto de estimulação o brincar possui um papel fundamental, pois é caracterizado como um complexo de ações criativas e comportamentos repletos de sentidos, que agrega valores no espaço e tempo em que a atividade é realizada. Brincando a criança se apropria de uma determinada cultura, passa por processo de transformação e contribui na construção do seu próprio desenvolvimento (TAKATORI M, 2012).

A brincadeira proporciona à criança a capacidade de conhecer a si a mesma, aprendendo a lidar com seus sentimentos e suas emoções. Auxilia no desenvolvimento da imaginação e criatividade, possibilitando a interação social, contribuindo na concepção da singularidade do outro e a experiência de perceber a necessidade de compreender o espaço e limitações que vão proporcionar melhora em suas relações sociais (dos SANTOS AJ, et al., 2017).

A família apresenta um papel muito importante para o desenvolvimento do brincar, pois representa o inicio da aprendizagem das relações sociais. As crianças são constantemente influenciadas pelos exemplos dos pais, pelo meio em que vivem, seus costumes, a cultura, classes sociais e etnias. Assim o homem é considerado como um ser eminentemente social e essa base familiar é extremamente importante para que a criança dê continuidade nesse processo de descobertas, principalmente através do brincar (PAPALIA ED e FELDMAN RD, 2013).

O brincar entre criança e cuidador favorece o fortalecimento do vínculo, e de relações sociais da criança com toda a família. O infantil passa a ter confiança para se expressar, seja através da linguagem verbal ou não verbal, com sinais e gestos. O papel do cuidador é tão necessário que é possível afirmar que os cuidadores ao brincarem, contribuem para a melhora no desenvolvimento de crianças com problemas de desenvolvimento (SOUZA DS, et al., 2017). 
Porém apesar das evidências sobre a importância do brincar, estudos apontam que a brincadeira perde seu importante papel, adquirindo baixa exploração pelos cuidadores. A tecnologia é inserida no universo das crianças, ocasionando, muitas vezes, redução de habilidades, ociosidade e sedentarismo, apresentando atrasos no desenvolvimento pela falta de exercícios de maneira mais ativa, não suprindo demandas motoras e cognitivas (RYMOVICZ MTP, 2013).

O brincar, neste contexto, é considerado como um instrumento eficaz e utilizado como recurso terapêutico para treino de atividades do cotidiano, promoção de habilidades psicomotoras globais, promovendo 0 desempenho ocupacional de crianças com atrasos ou déficits, favorecendo o desenvolvimento e viabilizando a aquisição de habilidades (JOAQUIM RHVT, et al., 2018; de CAMPOS SDF, et al., 2017).

Assim, tendo em vista a importância da brincadeira e do cuidador para o desenvolvimento infantil, esta pesquisa objetiva compreender a percepção do cuidador familiar acerca do brincar de crianças em vigilância do desenvolvimento.

\section{MÉTODOS}

Estudo de abordagem qualiquantitativa, de natureza descritiva-exploratória. A pesquisa foi realizada no laboratório do projeto de vigilância do desenvolvimento ACRESCER, da Escola Superior da Amazônia (ESAMAZ), em um município do estado do Pará. Foram critérios de inclusão: cuidadores familiares de crianças com $50 \%$ de frequência nos atendimentos no ACRESCER no período de abril à junho de 2018 , que assinaram o Termo de Consentimento Livre e Esclarecido (TCLE).

Foram critérios de exclusão: cuidadores que não aceitaram participar, crianças com frequência abaixo de $50 \%$ no período de abril à junho de 2018 , cuidadores não familiares e os que se recusaram a assinar o TCLE. Assim, apenas quatro cuidadores familiares de crianças com idade entre um ano e três meses a seis anos se enquadraram nos critérios.

O presente estudo foi aprovado pelo Comitê de Ética em Pesquisa da FACULDADE INTEGRADA BRASIL DA AMAZÔNIA - FIBRA, sob o número de aprovação do CAAE 98354718.6.0000.8187. Após aprovação foi realizado o contato com a coordenação do projeto ACRESCER, para a realização da consulta aos prontuários e em seguida contato com os cuidadores e assinatura do TCLE.

Todos que participaram da entrevista foram esclarecidos a respeito dos objetivos e participaram de forma voluntária. A identidade dos sujeitos foi mantida em sigilo, os participantes foram identificados como Cuidador A, B, C, D, e os nomes das crianças foram substituídos por pseudônimos.

Os autores desenvolveram uma entrevista semiestruturada, baseada no Histórico Lúdico de Takata que busca compreender a percepção do cuidador familiar de crianças em vigilância do desenvolvimento acerca do brincar, com questões socioeconômicas (escolaridade, renda familiar e etc..), além das que envolviam a percepção dos cuidadores sobre como: consideravam o brincar importante; com quem e como as crianças brincavam; qual a duração da brincadeira, entre outros (TAKATA N, 1971).

As entrevistas foram registradas em gravador de voz de celular, transcritas e analisadas. Os resultados foram organizados em quatro eixos temáticos que imergiram dos discursos dos pais, sendo utilizada para isso a técnica de análise de conteúdo, uma forma metodológica aplicada em discursos diversos (BARDIN L, 2011). Todas as etapas desta pesquisa foram organizadas em um fluxograma apresentado a seguir (Figura 1). 
Figura 1 - Fluxograma etapas da pesquisa.

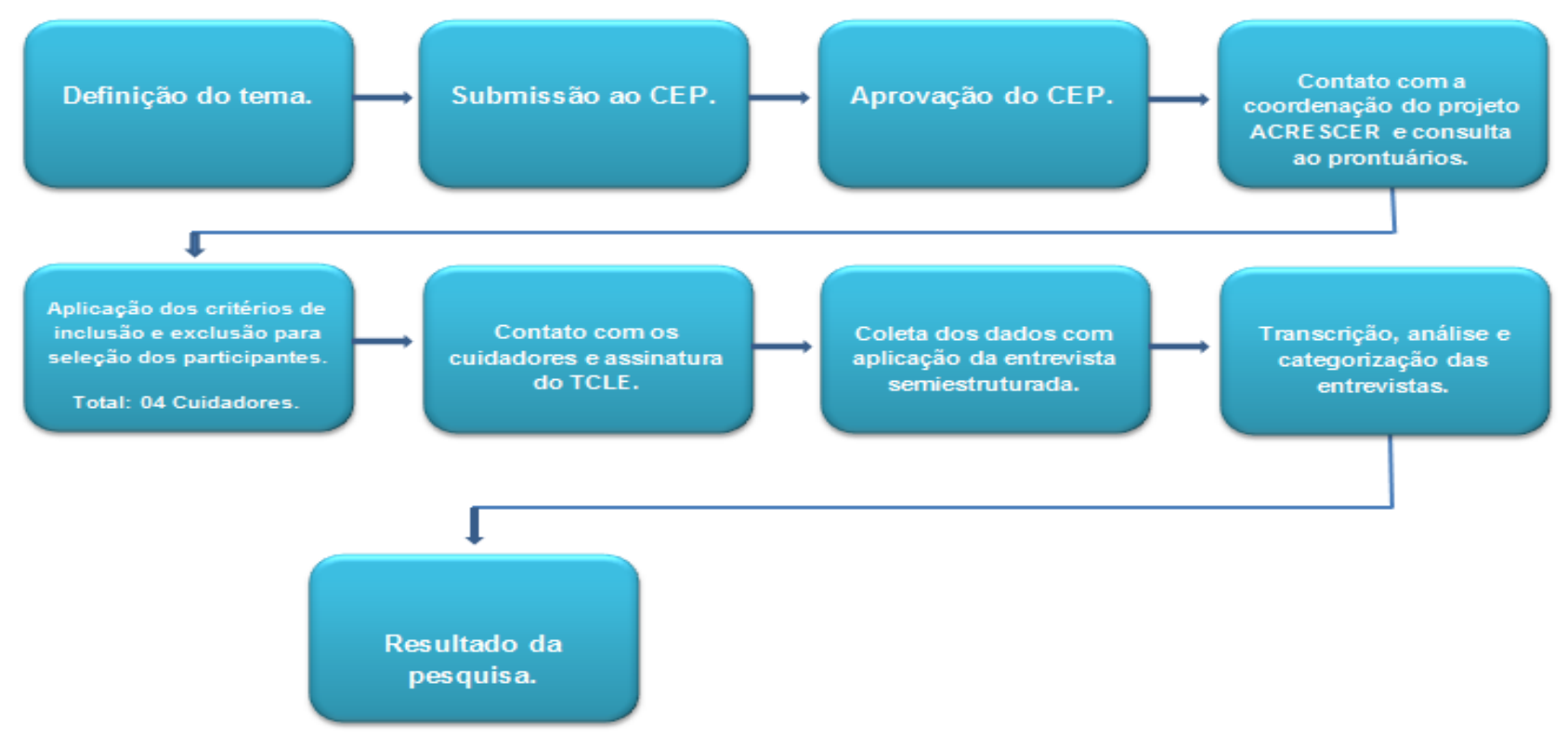

Fonte: Coimbra DC, et al., 2020.

\section{RESULTADOS E DISCUSSÃO}

Os resultados que emergiram da análise foram identificados e ordenados em quatro eixos temáticos sendo: $O$ tempo da brincadeira, $O$ brincar com outras crianças, $O$ brincar e o desenvolvimento da criança, $A$ participação do cuidador durante o brincar. A seguir serão apresentados os dados referentes a caracterização dos participantes quanto ao grau de parentesco, nível de escolaridade, idade das crianças e renda familiar (Tabela 1).

Quanto a caracterização dos participantes da pesquisa observa-se que: a maior ocorrência de cuidadores são mães, $75 \%$ dos entrevistados possuía uma renda acima de quatro salários-mínimos e também $75 \%$ apresentava como nível de escolaridade o ensino superior completo. Quanto a idade das crianças acompanhadas no projeto corresponde a uma faixa etária diversificada em um intervalo de um ano e seis meses à seis anos.

Tabela 1 - Caracterização dos participantes da pesquisa.

\begin{tabular}{lll}
\hline Variável & N & $\%$ \\
\hline Cuidador familiar & & $50 \%$ \\
\hline Mãe & 2 & $25 \%$ \\
Pai & 1 & $25 \%$ \\
Avó & 1 & $25 \%$ \\
\hline Idade da criança acompanhada & & $50 \%$ \\
\hline 1 ano e 6 meses & 1 & $25 \%$ \\
2 anos & 2 & \\
6 anos & 1 & $25 \%$ \\
\hline Renda familiar & & $25 \%$ \\
\hline 7 salários-mínimos & 1 & $25 \%$ \\
5 a 6 salários-mínimos & 1 & $25 \%$ \\
4 salários-mínimos & 1 & \\
1 a 2 salários-mínimos & 1 & $75 \%$ \\
\hline Escolaridade & & $25 \%$ \\
\hline Superior Completo & 3 & 2020.
\end{tabular}

Legenda: №- frequência absoluta; \%- percentagem. Fonte: Coimbra DC, et al., 2020. 
Dentre os aspectos levantados sobre os cuidadores ressalta-se a importância do nível de escolaridade. Estudos demonstram que a escolaridade da mãe impacta diretamente no desenvolvimento infantil de maneira positiva ou negativa. O maior nível de escolaridade pode estar relacionado ao maior conhecimento sobre os marcos do desenvolvimento, o que irá ajudar o cuidador a desenvolver ações que favoreçam a aquisição de habilidades, favorecendo satisfatoriamente o desenvolvimento da criança (ALVARENGA P, et al. 2020).

\section{O tempo da brincadeira}

$\mathrm{O}$ ato de brincar perpassa por várias gerações e encontra-se em todos os contextos que a criança vive ressaltando a importância da utilização de recursos que possam desenvolver o crescimento e o potencial mental de cada uma. Proporcionando ao brincante a capacidade de conhecer a si mesma, aprender a lidar com seus sentimentos e suas emoções, e a explorar seu poder de imaginação e criatividade. Por meio da prática do brincar que as crianças tendem a desenvolver suas experiências de vida, as descobertas e a realização da construção da personalidade (SILVA AFF e SANTOS ECM, 2009; PAIXÃO AB, et al., 2016).

Os adultos são fomentadores da brincadeira, cabe a ele a construção do tempo do brincar onde propiciam os espaços, objetos e estrutura necessária para a sua realização, fazendo-se presente na mediação e partilha em um dos principais estímulos para o desenvolvimento infantil. Valorizando o tempo do brincar como uma ação livre e ressignificada, assumindo uma função etica de direcionamento ou partilha, transmitindo segurança e conhecimentos (BARBOSA MCS, 2013).

Os relatos demonstram que as crianças passam a maior parte do tempo livre brincando, ou em atividades de lazer, como podemos analisar nos discursos dos cuidadores A, B e C:

"De manhã umas 10h que a gente desce pra ele correr [..]a partir das 4 h da tarde a gente desce e só sobe $8 h$ " (Cuidador A)

"Da hora que acorda, até a hora de dormir. Não, não tem um tempo limite[..]" (Cuidador B)

"Costuma brincar pela parte da manhã, [...] 4horas e parte da tarde [...] 3 horas." (Cuidador C)

Não se identificou interferências significativas para a realização da atividade de brincar, sendo este, inclusive, incentivado e facilitado pelos cuidadores. Analisa-se com o diálogo dos cuidadores $A, B$ e $C$ que $o$ brincar é o principal fator de ocupação da criança. Os cuidadores $A$ e $C$ conseguem mensurar horários que a brincadeira ocorre com maior frequência, enquanto que o cuidador $B$, não determina um tempo, uma vez que a criança possui liberdade e autonomia para brincar.

O cuidador D enfatiza não haver um tempo determinado para o brincar, salvo o horário da escola e do descanso, a criança passa o restante do tempo brincando.

"Depois que ela chega da escola toma banho, almoça, aí ela brinca, dorme e depois quando ela acorda umas 17 h ela brinca de novo." (Cuidador D)

O tempo para as brincadeiras não deve ser limitado. Os pais devem fazer poucas interferências, permitindo que esteja a critério da criança a duração dessa atividade, proporcionando uma maior autonomia e independência e proporcionando uma liberdade às tomadas de decisões (NUNES FBS, et al., 2013). Questionou-se durante a entrevista a opinião dos cuidadores sobre o tempo do brincar, se consideravam suficiente. O cuidador A não respondeu a pergunta. Percebe-se por meio do discurso do cuidador $\mathrm{B}$ a dedicação e disponibilidade de tempo para brincar com a criança:

"[...] além dele se distrair muito, ele brinca constante comigo [...] eu quase não consigo fazer nada porque pra onde eu vou ele vai junto e leva os brinquedos [...] $A$ gente passa o dia inteiro assim [...]." (Cuidador B)

Esta afirmação pode ser analisada a partir do momento que o cuidador B relata que não há período estipulado para a brincadeira, ela torna-se livre e espontânea. 
Identifica-se que todos os cuidadores familiares consideram suficiente o tempo de duração do brincar, como podemos observer no discurso dos cuidadores $\mathrm{C}$ e D.

"Considero suficiente. Porque é o tempo que ele tá acordado sem realizar outas atividades." (Cuidador C)

"[...] Considero porque em casa ela brinca bastante e aprende com isso [...]." (Cuidador D)

Explorando os discursos observamos que os cuidadores enfatizam o importante papel do brincar para o desenvolvimento da criança, favorecendo a interação e a aprendizagem e reconhecendo-o como ocupação principal da criança.

Pedroso MCS (2013) e Lameirão L (2015) ressaltam que por meio do brincar a criança desenvolve aspectos cognitivos, potencializa seu desempenho sensório-motor e desenvolve habilidades sociais e culturais, facilitando no processo educacional e no ganho de valores fundamentais a necessidade humana.

\section{O brincar com outras crianças}

Em relação a esta temática, pode-se observar a compreensão dos cuidadores familiares quanto à interação social da criança através do brincar. Todos destacaram que compreendem esse momento como um facilitador para o desenvolvimento das relações sociais. A criança inicia o brincar, desde o seu nascimento, onde esta atividade do sujeito é praticamente desenvolvida no ambiente familiar, tornando a brincadeira uma espécie de elo comunicativo entre o brincante e a família, favorecendo o convívio social, passando ser o primeiro conceito de sociedade para ela (KISHIMOTO TM, 2011).

Para os cuidadores $\mathrm{A}$ e $\mathrm{B}$, esse momento permite que as crianças realizem boas relações sociais com outros indivíduos da mesma idade, de maneira que isso some ganhos positivos para a interação social.

"Com certeza. Pra interagir [...]." (Cuidador A)

"Sim, quando chega criança em casa ele pira (risos), porque ele desenvolve né, ele desenvolve mais ainda, a convivência, a interação com outra criança [...]" (cuidador B)

O cuidador $C$ e cuidador $D$ enfatizam a interação com outras crianças como um momento de aprendizado, trocas de experiências e possibilidades de vivenciar atividades com indivíduos da mesma faixa etária, sem interferência de adultos.

"[..] Considero, acho que é uma fase de aprendizado, uma questão de intermediar interesses e conflitos [...] acho que a criança aprende muito com essa intermediação do outro." (Cuidador $C$ )

"[...] Considero. Porque senão, ela fica só com os adultos no caso, a minha família basicamente é toda adulta [...] ai ela já vai brincar com a Mariazinha e o Gustavinho que é da rua dela pra ela socializar, senão ela vai ficar só nesse mundo que a gente vive." (Cuidador D)

A interação social entre crianças influência na percepção sobre si mesma e sua interação com o ambiente, a formação de atitudes sociais, seguimento de regras e responsabilidade mediante o seu contexto familiar, 0 repasse cultural e o aprendizado oriundo das vivências escolares e de outras ocupações. A brincadeira possibilita a concepção da singularidade do outro desenvolvendo seus contextos de aceitação e a experiência de perceber a necessidade de compreender o espaço e limitações que vão proporcionar melhora em suas relações sociais (MEDEIROS CCM, et al., 2019; de LIMA CA, et al., 2014).

\section{O brincar e o desenvolvimento da criança}

Através do discurso dos cuidadores identificou-se que eles compreendem o brincar como fator potencializador do desenvolvimento, reforçando o aprendizado. O brincar foi identificado como um 
instrumento de comunicação entre cuidador e criança, otimizador de autonomia para tomada de decisões repercutindo nas vivências da brincadeira. Podendo ser observado no discurso do cuidador A e B:

"[...] Faz ele aprender a dividir, aprender a mostrar as coisas pra mim, até porque ele ainda não fala, então quando ele brincar eu tento ensinar ele o nome dos brinquedinhos é aprendizado na brincadeira." (Cuidador A)

"[...] Na brincadeira que ele tá aprendendo [...] ele aprendeu algumas cores, vê nos desenhos, nas brincadeiras, tudo que acontece lá ele traz pro dia a dia e vai aprendendo." (Cuidador B)

O brincar proporciona diretamente a estimulação do desenvolvimento neurobiológico, além do favorecimento de uma experiência social que adere valores culturais que capacitam a criança para 0 enfrentamento de vivências futuras, permitindo testar noções das particularidades dos adultos, a comunicação da criança com o meio e projetando a busca de novos desafios. Com o brincar a criança aprende a ter domínio de si e do ambiente, tornando um momento significativo no processo de construção da infância como fator potencializador para o desenvolvimento (de SOUZA AJ, 2020).

Para o cuidador A e B a brincadeira passa a ter um significado no aprendizado por meio da reprodução e aumento no repertório de palavras e figuras, ressaltando um desenvolvimento mais acelerado através do lúdico. O brincar se faz importante em todos os aspectos do desenvolvimento, seja para habilidades sociais, seja como instrumento dos educadores, atuando como facilitador no processo de aprendizagem. A ludicidade contribui para o processo, tornando as atividades mais atraentes e menos cansativas, potencializando aspectos cognitivos como atenção e concentração (GASPAR VSA, 2020). O cuidador C menciona no discurso que a brincadeira contribui na aprendizagem e no processo de autonomia e dos estabelecimentos dos limites que a criança pode ter:

"[...] autonomia, do aprendizado, do fazer e saber o limite que a criança pode ter." (Cuidador C)

O cuidador $\mathrm{D}$, fez uma comparação interessante ao relatar a superproteção de outros pais, onde nota-se que a outra criança mencionada já apresenta dificuldades para estabelecer interações sociais.

"[...] eu faço comparação com o sobrinho que eu tenho, que a mãe dele não deixa ele fazer nada, superproteção, não põe ele no chão, não senta com ele pra brincar e a gente tá percebendo que ele tá muito introvertido [...] Ele não desenvolve nessa área social." (Cuidador D)

Para a Terapia Ocupacional o brincar assume papel importantíssimo para a atuação do profissional, considerando favorecer habilidades singulares da criança, sejam de aspectos motores, sociais e cognitivos. Tendo em vista o brincar como sua principal ocupação, facilitador no engajamento e interesse de participação da criança (AOTA, 2015).

\section{A participação do cuidador durante o brincar}

A maneira como o cuidador vai estar incluso nas vivências da criança varia de acordo com o contexto familiar ao qual estão inseridos. Toda ação realizada pelo adulto irá influenciar no comportamento e desenvolvimento da criança, sendo assim, é dever do cuidador estruturar essa vivência de maneira que a sua participação gere ensinamentos, organização e fortalecimento do elo cuidador e criança (MONDIN EMC, 2005). Outro aspecto que irá influenciar na participação do cuidador é a cultura, pois a partir das condutas dos pais, poderá moldar os costumes das crianças influenciando no seu brincar, no qual reproduzem aquilo que está em seu convívio, sendo de fundamental importância para o processo a participação do cuidador, agregando valores sociais e culturais, para que a criança possa expressar-se de acordo com suas próprias ideias e sentimentos (PASTORE MN e BARROS DD, 2015).

O brincar aparece nestas narrativas como fundamental para o desenvolvimento e a construção de saberes e conhecimentos, agregados a valores culturais e sociais, além das responsabilizações e relações que as 
crianças estabelecem entre pares e com os adultos. Os cuidadores relataram assumir papéis diferentes durante as brincadeiras com as crianças. Alguns participam ativamente do brincar, enquanto outros atuam como mediadores ou observadores do processo.

"Olha, eu acho que é muito importante a criança ter contato de brincadeira com a mãe, [...] tem que sentar e brincar com a criança." (Cuidador A)

"Eu acho ótimo, inclusive assim eu amo meu trabalho por conta disso, porque ele tem essa flexibilidade entendeu? [...] ele fica tão empolgado de querer tá brincando, de querer tá interagindo com a gente [...]." (Cuidador $B$ )

"A minha participação? Eu acho que vai mais como um observador, digamos assim, mais como um orientador também [...]." (Cuidador C)

Por meio do relato dos cuidadores, é possível perceber que as crianças iniciam o brincar sozinhas, mas buscam de maneira espontânea a interação com o cuidador familiar, para compartilhar a brincadeira ou para solicitar um auxílio.

"Eu acho que é muito importante [...] ela já tem uma noção do que é, e ela já chama os pais para brincar quando estão em casa, [...] eu acho que essa parte é muito importante, tá com alguém orientando as ideias dela, tipo fazendo acolhimento do que ela tá pensando e direcionando, sabe?" (Cuidador D)

O brincar é a ocupação principal da criança e precisa ser explorado e vivenciado, pois interfere diretamente na qualidade de vida e no processo de amadurecimento e aprendizagem. Proporcionar o brincar, é permitir que a criança se perceba como um ser único e complexo, com suas próprias preferências, opiniões e ideologias. O brincar é um potencializador do desenvolvimento infantil em suas mais diversas áreas e o brincar entre 0 adulto e a criança, permite que esta expresse seus sentimentos mais facilmente, de maneira que tenha seja mais claro para ela o controle de suas emoções (BRASIL, 2017).

Constata-se, nos discursos, que os cuidadores familiares apresentam argumentos concisos, mostrando clareza sobre as funções do brincar para o desenvolvimento infantil, possuem um esclarecimento sobre a importância do brincar no universo infantil. Sendo assim, os cuidadores familiares também entendem o brincar como principal ocupação da criança, e que participar dessa atividade, favorece o vínculo familiar, prepara a criança para compreender as relações sociais e quando há interação com outras crianças geram laços externos, possibilitando assim, que ela assuma sua identidade diante da sociedade.

\section{CONCLUSÃO}

Os cuidadores familiares apresentam a percepção do brincar como fonte importante para o desenvolvimento infantil, são capazes de identificar os benefícios da brincadeira para o processo de aprendizagem e para o desenvolvimento de relações sociais, ressaltando que o tempo diário para brincar deve ser livre e com pouca interferência dos adultos na escolha do brincar, porém para a maioria dos cuidadores, o adulto deve ser ativo durante a brincadeira. Acredita-se que seja de alta relevância estudos sobre a temática, visto que o cuidador principal é quem passa maior parte do tempo com a criança, sendo necessária sua compreensão acerca da importância no aproveitamento deste processo. Neste contexto, acredita-se que este trabalho possa dar subsídios para o surgimento de novas pesquisas na área, incentivando novos estudos dirigidos sobre os processos de intervenções com cuidadores e assim, proporcionar a comunidade acadêmica interessada no assunto, uma visão do cuidador familiar acerca do brincar das crianças sob vigilância do desenvolvimento.

\section{REFERÊNCIAS}

1. ALVARENGA $P$, et al. Escolaridade materna e indicadores desenvolvimentais na criança: mediação do conhecimento materno sobre o desenvolvimento infantil. Psico, Porto Alegre, 2020; 51 (1): 1-14. 
2. American Occupational Therapy Association. Estrutura da prática da Terapia Ocupacional: domínio \& processo - 3ª ed. traduzida. Rev. Ter. Ocup. Univ. São Paulo, 2015; 26(esp): 1-49.

3. BARBOSA MCS. Tempo e cotidiano- tempos para viver a infância. Leitura: Teoria \& Prática, Campinas, 2013 ; 31 (61): 213-222.

4. BARDIN L. Análise de Conteúdo. Tradução de Luís Antero Reto, Augusto Pinheiro: São Paulo, 2011; 279 p.

5. BRASIL. 2017. In: Ministério da Educação. Brasília: Ministério da Educação. Disponível em: http://basenacionalcomum.mec.gov.br/images/BNCC_El_EF_110518_versaofinal_site.pdf. Acesso em: 5 mar. 2020.

6. de CAMPOS SDF, et al. O brincar para o desenvolvimento do esquema corporal, orientação espacial e temporal: análise de uma intervenção. Cad. Bras. Terap. Ocup. UFSCAR, São Carlos, 2017; 25(2): 275-285.

7. de LIMA CA, et al. A importância do brincar para a criança. Revista Científica Instituto Saber de Ciências IntegradasSinop/MT, Brasília, 2014; 2(1): 165.

8. de SOUZA AJ. Considerações sobre o brincar: porque os brinquedos auxiliam e podem influenciar nos processos de aprendizado. Revista Acervo Educacional, 2020; 2: 2126.

9. dos SANTOS AJ, et al. A Percepção De Crianças E Cuidadores Sobre A Importância Do Brincar Espontâneo Para O Desenvolvimento Infantil. XVI SEPA - Seminário Estudantil de Produção Acadêmica. Universidade SalvadorUNIFACS, Salvador, 2017; 16: 246-262.

10. FIGUEIRAS AC, et al. Manual para vigilância do desenvolvimento infantil no contexto da AIDPI. Washington: DC: OPAS, 2005; $54 \mathrm{p}$.

11. GASPAR VS de A. O LÚDICO COMO INSTRUMENTO DE ALFABETIZAÇÃO. Revista Artigos. Com., $2020 ; 15: 2574$.

12. JOAQUIM RHVT, et al. O faz de conta e as brincadeiras como estratégia de intervenção para uma criança com atraso no desenvolvimento infantil. Cad. Bras. Ter. Ocup. UFSCAR, São Carlos, 2018; 26(1): 63-71.

13. KISHIMOTO TM. O brincar e suas teorias. São Paulo: Cengage Learning, 2011; 172p.

14. LAMEIRÃO L. Panorama das conquistas da criança durante a primeira infância. Arte Médica Ampliada. 2015; 35(2): 64-70.

15. MEDEIROS CCM, et al. Transcendendo o problema: percepções de mães e crianças sobre o impacto do transtorno do desenvolvimento da coordenação no dia a dia. Cad. Bras. Ter. Ocup. UFSCAR, São Carlos, $2019 ; 27$ (4): $792-$ 805.

16. MONDIN EMC. Um olhar ecológico da familia sobre o desenvolvimento humano. Psicologia Argumento, Curitiba, 2005; 23 (41): 25-35.

17. NUNES FBS, et al. Retratos do cotidiano de meninos de cinco e seis anos: a atividade de brincar. Cad. Bras.Ter. Ocup. UFSCar, São Carlos, 2013; 21(2): 275-287.

18. PAIXAO AB, et al. Importância das atividades lúdicas na terapia oncológica infantil. Cuidarte Enfermagem. 2016; 10(2): 209-216.

19. PAPALIA ED, FELDMAN RD. Desenvolvimento humano. 12 ed. Porto Alegre: AMGH, 2013; 793p.

20. PASTORE MN, BARROS DD. A cultura do brincar e a socialização infantile: percepções sobre o ser criança numa comunidade moçambicana. Cad. Bras. de Ter. Ocup. UFSCar, São Carlos, 2015, 23(3): 599-609.

21. PEDROSO MCS. A Função do Brincar para a Criança com Deficiência. Revista Científica da FHO UNIARARAS, Araras, 2013; 2(1): 82-925.

22. RYMOVICZ MTP. O Brincar Na Educação Infantil: A Compreensão de Pais das Crianças de 3 Anos do CMEI Pedacinho do Céu De São Mateus Do Sul - Paraná. Monografia (Especialização em docência na educação infantil) - Núcleo de Estudos e Pesquisas em Infância e Educação Infantil da Universidade Federal do Paraná, Curitiba, 2013; $31 \mathrm{p}$.

23. SILVA AFF, SANTOS ECM. A Importância Do Brincar Na Educação Infantil. Monogrfia (Especialização Desafios Do Trabalho Cotidiano: A Educação das Crianças de 0 A 10 Anos") - Decanato de Pesquisa e Pós-Graduação - DPPG da Universidade Federal Rural Do Rio De Janeiro - UFRRJ, Mesquita, 2009; 36 p.

24. SOUZA DS, et al. O brincar de crianças com deficiência física sob a perspectiva dos pais. Cad. Bras. Ter. Ocup. UFSCAR, São Carlos, 2017; 25 (2): 267-274.

25. TAKATA N. The play milieu-a preliminary appraisal. American Journal of Occupational Therapy, 1971; 25: $281-284$.

26. TAKATORI M. O brincar na terapia ocupacional: um enfoque na criança com lesões neurológicas. São Paulo: Zagodoni, 2012; 176p. 